\title{
Surface Wave Characteristics at the Interface of Welded Elastic Halfspaces
}

\author{
J. N. Sharma ${ }^{1}$, K. K. Sharma², Ashwani Kumar² \\ ${ }^{1}$ Department of Mathematics, National Institute of Technology, Hamirpur, India; \\ ${ }^{2}$ Department of Physics, National Institute of Technology,Hamirpur, India. \\ Email: jns@nitham.ac.in, kks@nitham.ac.in, puri_nit@yahoo.com \\ Received June $1^{\text {st }}$, 2011; revised June 20 $0^{\text {th }}$, 2011; accepted June $25^{\text {th }}, 2011$.
}

\begin{abstract}
The present article concentrates on the propagation of generalized surface acoustic waves in a composite structure consisting of piezoelectric and non-piezoelectric semiconductor media. The mathematical model of the problem is depicted by a set of partial differential equations of motion, Gauss equation in piezoelectric and electron diffusion equation in semiconductor along with boundary conditions to be satisfied at the interface. The secular equation that governs the propagation of surface waves has been derived in compact form after obtaining the formal solution. The analytic expressions for displacements, stresses, piezoelectric potential and electron concentration during the surface wave propagation at the interface have also been obtained. The numerical solution of the secular equation is carried out for the cadmium selenide and silicon composite by employing fixed point functional iteration numerical method along with irreducible Cardano method. The computer simulated results with the help of MATLAB software in respect of dispersion curves, attenuation coefficient, displacements, stresses, carrier concentration and piezoelectric potential are presented graphically. This work may be useful in surface acoustic wave (SAW) devices and electronic industry.
\end{abstract}

Keywords: Piezoelectrics, Life Time, Silicon, Dispersive Waves, Attenuation

\section{Introduction}

The piezoelectric effect in certain noncentro-symmetric crystalline materials was discovered by Curie and Curie (1880). Parmenter (1953) regarded the appearance of DC electric field along the direction of propagation of a acoustic wave in a medium containing mobile charges as acoustoelectric effect. Weinreich et al. (1959) termed acoustoelectric effect as wave particle drag phenomenon. Hutson and White (1962) found that the field produced along the traveling acoustic wave produces current and space charges which results in acoustic dispersion and loss. According to White (1962) an acoustic wave traveling in a piezoelectric semiconductor can be amplified or attenuated by the application of a DC electric field. Collins et al. (1968) found the strong interaction between the wave on the surface of piezoelectric crystal and the wave on the drifting carriers in a nearby semiconductor. Bluestein (1968) and Gulyaev (1969) studied surface acoustic waves in piezoelectric materials. Fischler (1970) proposed that acoustoelectric amplification can be better obtained in composite structure of semiconductor and piezoelectric materials. Dietz et al. (1988) explored that the acoustoelectric amplification of acoustic waves can also be achieved through composite of a piezoelectric dielectric and non-piezoelectric semiconductor.

de Lorenzi and Tierten (1975), and Maugin and Dehar (1986) developed nonlinear theories for deformable semiconductors. Ingebrigtsen (1970) studied linear and non-linear attenuation of acoustic surface waves in a piezoelectric coated with a semiconductor film. Tien (1968) presented the nonlinear theory of ultrasonic amplification and current saturation in piezoelectric semiconductors. Kagan (1997) investigated the surface wave propagation in a piezoelectric crystal underlying a two dimensional conducting layer. Jin et al. (2002) studied the Lamb wave propagation in a metallic semi-infinite medium covered with piezoelectric layer. Wang (2002) investigated wave propagation in the piezoelectric solid medium. Yang and Zhou (2005) investigated amplification of acoustic waves in piezoelectric semiconductor plates. Yang and Zhou (2005) also studied the propagation and amplification of gap waves between a piezoelectric halfspace and a semiconductor film. Maruszewski (1989) considered the interactions between elastic, thermal and charge carrier's fields in semiconductors and predicted the existence of two kinds of waves namely, polarized and dispersive waves. Kleinert et al. (2005) studied the surface-acoustic-waveinduced space-charge waves in electron-hole systems.

Sharma and Pal (2004) investigated the propagation of Lamb waves in homogeneous, transversely isotropic, piezothermoelastic plate. Sharma et al. (2005) studied the propagation characteristics of Rayleigh waves in transversely isotropic piezothermoelastic materials. The phase velocity profiles are found to be dispersive at small values of wave number and these become asymptotically linear at higher values of wave numbers. Sharma and Walia (2007) carried out further investigations on the propagation of Rayleigh waves in a homogeneous, transversely isotropic, piezothermoelastic semi-space. Sharma and Thakur (2006) studied the plane harmonic elasto-thermodiffusive waves in semiconductor materials. Sharma et al. (2007, 2009) also investigated the characteristics of elasto-thermodiffusive wave propagation on semiconductor materials and observed that life time of charge carriers and thermal relaxation time affects the wave characteristics significantly at long wavelengths as compared to that at short wavelengths. Sharma et al. (2008) investigated the elasto-thermodiffusive surface 
waves in a semiconductor halfspace underlying a fluid with varying temperature. Recently, Sharma et al. (2010) studied the surface waves at the interface of semiconductor layer over a piezoelectric halfspace and found that phase velocity as well as attenuation decreases with the decreasing life time of the carrier field.

Keeping in view the above work, the present article is devoted to give detailed information of generalized surface acoustic waves at the interface of the piezoelectric and semiconductor halfspaces. The behavior of displacement components, stresses, electron concentration and piezoelectric potential at the interface of considered structure has been discussed. The effect of life time of the carrier field on phase velocity and attenuation coefficient is also taken into consideration so as to understand the interaction of acoustic wave in the piezoelectric halfspace with the carriers in the semiconductor halfspace.

\section{Formulation of Problem}

We consider a composite structure consisting of a homogeneous transversely isotropic piezoelectric halfspace and a homogeneous isotropic, non-piezoelectric elastic semiconductor halfspace which are in welded contact with each other as shown in Figure 1. We take the origin of coordinate system oxyz at any point on the plane surface (interface) and z-axis pointing vertically downward into the piezoelectric halfspace along the poling direction. Thus, the piezoelectric halfspace and the semiconductor medium are represented by $z \geq 0$ and $z \leq 0$ respectively. We choose $x$-axis along the direction of wave propagation in such a way that all particles on a line parallel to the $y$-axis are equally displaced. Therefore, all field quantities are independent of y-coordinate.

Further, the disturbance is assumed to be confined in the neighborhood of the interface $(z=0)$ and hence vanishes as $z \rightarrow \infty$. The basic governing equations of motion and electron diffusion for the composite structure under study, in the absence of body forces and electric sources, are given below:

1) Homogeneous isotropic, $n$-type semiconductor elastic halfspace [Maruszewski (1989), Sharma et al. (2007)]:

$$
\begin{gathered}
\mu \nabla^{2} \boldsymbol{u}^{s}+(\lambda+\mu) \nabla \nabla \cdot \boldsymbol{u}^{s}-\lambda^{n} \nabla N=\rho^{s} \ddot{\boldsymbol{u}}^{s} \\
\rho^{s} D^{n} \nabla^{2} N-\rho^{s}\left(1+t^{n} \frac{\partial}{\partial t}\right) \dot{N}-a_{2}^{n} T_{0} \lambda^{T} \nabla \cdot \dot{\boldsymbol{u}}^{s}=-\left(1+t^{n} \frac{\partial}{\partial t}\right)\left(\frac{\rho^{s}}{t_{n}^{+}}\right) N
\end{gathered}
$$

2) Homogeneous, transversely isotropic, piezoelectric (6 mm

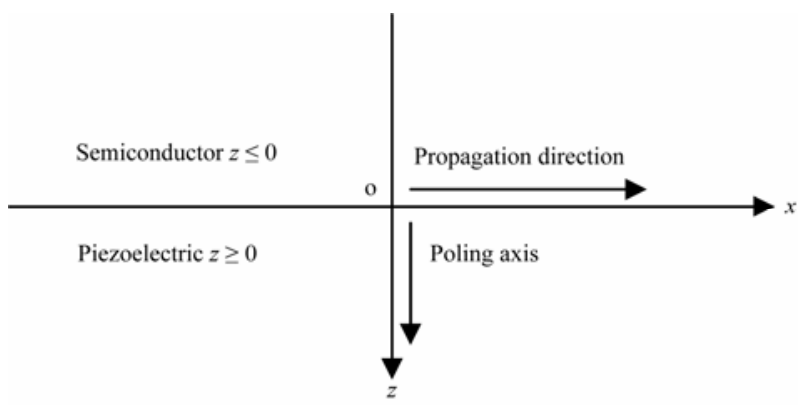

Figure 1.

Geometry of the problem. class) medium [Sharma and Pal (2004)]:

$$
\begin{gathered}
c_{11} u_{, x x}^{p}+c_{44} u_{, z z}^{p}+\left(c_{13}+c_{44}\right) w_{, x z}^{p}+\left(e_{15}+e_{31}\right) \phi_{, x z}^{p}=\rho^{p} \ddot{u}^{p} \\
\left(c_{13}+c_{44}\right) u_{, x z}^{p}+c_{44} w_{, x x}^{p}+c_{33} w_{, z z}^{p}+e_{15} \phi_{, x x}^{p}+e_{33} \phi_{, z z}^{p}=\rho^{p} \ddot{w}^{p} \\
\left(e_{15}+e_{31}\right) u_{, x z}^{p}+e_{15} w_{, x x}^{p}+e_{33} w_{, z z}^{p}-\varepsilon_{11} \phi_{, x x}^{p}-\varepsilon_{33} \phi_{, z z}^{p}=0
\end{gathered}
$$

where the notations $\nabla^{2}=\frac{\partial^{2}}{\partial x^{2}}+\frac{\partial^{2}}{\partial z^{2}}, \quad N=n-n_{0}, \quad a_{2}^{n}=\frac{a^{Q p}}{a^{Q}}$, $\lambda^{T}=(3 \lambda+2 \mu) \alpha_{T}$ have been used. In the above equations the superposed dots on various quantities denote time differentiation and comma nota- tion is used for spatial derivatives. Here $\lambda, \mu$ are Lamè's parameters; $\rho^{s}$ is the density; $\lambda^{n}$ is the elastodiffusive con- stants of electrons; $D^{n}$ is the diffusion coefficient of electron; $t_{n}^{+}$and $t^{n}$ are the life time and relaxation time of the carriers fields; $n_{0}$ and $n$ are the equilibrium and non-equilibrium values of electrons concentration; $\alpha_{T}$ is the coefficient of linear thermal expansion of the semiconductor material. The quantities $a^{Q p}, a^{Q}$ are flux-like constants and $T_{0}$ is the uniform temperature; $\boldsymbol{u}^{s}=\left(u^{s}, 0, w^{s}\right)$ and $\boldsymbol{u}^{p}=\left(u^{p}, 0, w^{p}\right)$ are displacement vectors for semiconductor and of piezoelectric materials respectively. The quantities $\phi^{p}, \rho^{p}, c_{i j}$ and $e_{i j}$ are the electric potential, density, elastic parameters and piezoelectric constants; $\varepsilon_{11}$ and $\varepsilon_{33}$ are the electric permittivity perpendicular and along the axis of symmetry of piezoelectric material, respectively. Throughout this paper the superscripts $p, s$ on the field quantities and material parameters refers to piezoelectric and semiconductor materials respectively.

The non-vanishing components of stresses, current density and electric displacement in both the media are:

$$
\begin{aligned}
\tau_{z z}^{s} & =(\lambda+2 \mu) \frac{\partial w^{s}}{\partial z}+\lambda \frac{\partial u^{s}}{\partial x}-\lambda^{n} N, \\
\tau_{x z}^{s} & =\mu\left(\frac{\partial u^{s}}{\partial z}+\frac{\partial w^{s}}{\partial x}\right), \\
J_{z}^{s} & =-e D^{n} N_{, z} \\
\tau_{z z}^{p} & =c_{13} \frac{\partial u^{p}}{\partial x}+c_{33} \frac{\partial w^{p}}{\partial z}+e_{33} \frac{\partial \phi^{p}}{\partial z}, \\
\tau_{x z}^{p} & =\frac{c_{44}}{2}\left(\frac{\partial u^{p}}{\partial z}+\frac{\partial w^{p}}{\partial x}\right)+e_{15} \frac{\partial \phi^{p}}{\partial x}, \\
D_{z}^{p} & =e_{31} \frac{\partial u^{p}}{\partial x}+e_{33} \frac{\partial w^{p}}{\partial z}-\varepsilon_{33} \frac{\partial \phi^{p}}{\partial z}
\end{aligned}
$$

Here $\tau_{i j}^{s}$ and $\tau_{i j}^{p}$ are the stress tensors. The quantities $J_{z}^{s}$ and $N_{z}$ respectively denote the current density and carrier density gradient in semiconductor; $D_{z}^{p}$ is the electric displacement vector of piezoelectric material and $e$ is the electronic charge. The above model consisting of partial differential equations of motion, Gauss equation and equation for electron diffusion is also subjected to the continuity of stresses, displacements, electric fields and current density at the interface $(z=0)$ of two media. Mathematically, this requirement leads to the following interfacial boundary conditions:

$$
\tau_{z z}^{p}=\tau_{z z}^{s}, \tau_{x z}^{p}=\tau_{x z}^{s}, u^{p}=u^{s}, w^{p}=w^{s}, \phi_{z}^{p}=N, D_{z}^{p}=J_{z}^{s}
$$

We define the following quantities 


$$
\begin{aligned}
& x^{\prime}=\frac{\omega^{*} x}{v_{l}}, z^{\prime}=\frac{\omega^{*} z}{v_{l}}, t^{\prime}=\omega^{*} t, t^{n^{\prime}}=\omega^{*} t^{n}, t_{n}^{t^{\prime}}=\omega^{*} t_{n}^{+}, \\
& N^{\prime}=\frac{N}{n_{0}}, D_{z}^{p^{\prime}}=\frac{\rho^{s} v_{l}^{2}}{\lambda^{n} n_{0} e_{33}} D_{z}^{p}, u^{s^{\prime}}=\frac{\rho^{s} \omega^{*} v_{l}}{\lambda^{n} n_{0}} u^{s}, \\
& w^{s^{\prime}}=\frac{\rho^{s} \omega^{*} v_{l}}{\lambda^{n} n_{0}} w^{s}, u^{p^{\prime}}=\frac{\rho^{s} \omega^{*} v_{l}}{\lambda^{n} n_{0}} u^{p}, w^{p^{\prime}}=\frac{\rho^{s} \omega^{*} v_{l}}{\lambda^{n} n_{0}} w^{p}, \\
& \tau_{i j}^{p^{\prime}}=\frac{\tau_{i j}^{p}}{\lambda^{n} n_{0}}, \tau_{i j}^{s^{\prime}}=\frac{\tau_{i j}^{s}}{\lambda^{n} n_{0}}, J_{z}^{s^{\prime}}=\frac{J_{z}^{s}}{e n_{0} v_{l}}, c_{1}=\frac{c_{33}}{c_{11}}, \\
& c_{2}=\frac{c_{44}}{c_{11}}, c_{3}=\frac{c_{13}+c_{44}}{c_{11}}, e_{1}=\frac{e_{15}+e_{31}}{e_{33}}, v_{l}^{2}=\frac{\lambda+2 \mu}{\rho^{s}}, \\
& v_{p}=\sqrt{\frac{e_{15}}{\rho_{33}}, \bar{\varepsilon}}, \bar{\rho}=\frac{\varepsilon_{11}}{\varepsilon_{33}}, \eta_{3}=\frac{\varepsilon_{33} c_{11}}{e_{33}^{2}}, \omega^{\prime}=\frac{\omega}{\rho^{*}}, c^{\prime}=\frac{c}{v_{l}} \\
& \phi^{p^{\prime}}=\frac{\lambda_{p} n_{0}}{\lambda^{T} T_{0}}, \delta_{1}^{2}=\frac{v_{l}^{2}}{v_{p}^{2}} \\
& \omega^{*}=\frac{v_{l}^{2}}{D^{n}}, \delta_{p}=\frac{e_{33} \omega^{*} \rho^{s} v_{l}}{c_{11} \lambda^{n} n_{0}}, \varepsilon_{n}=\frac{v_{t}^{2}}{v_{l}^{2}}, v_{t}^{2}=\frac{\mu}{\rho^{s}(\lambda+2 \mu) n_{0}}
\end{aligned}
$$

where $\omega^{*}$ is the characteristic frequency, and $v_{l}, v_{t}$ are respectively, the longitudinal and shear wave velocities. Upon introducing the quantities (9) in Equations (1) to (5) we obtain

$$
\begin{gathered}
\delta^{2} \nabla^{2} \boldsymbol{u}^{s}+\left(1-\delta^{2}\right) \nabla \nabla \cdot \boldsymbol{u}^{s}-\nabla N=\ddot{\boldsymbol{u}}^{s} \\
\nabla^{2} N-\left[-\frac{1}{t_{n}^{+}}+\left(1-\frac{t^{n}}{t_{n}^{+}}\right) \frac{\partial}{\partial t}+t^{n} \frac{\partial^{2}}{\partial t^{2}}\right] N-\varepsilon_{n} \nabla \cdot \dot{\boldsymbol{u}}^{s}=0 \\
u_{, x x}^{p}+c_{2} u_{, z z}^{p}+c_{3} w_{, x z}^{p}+e_{1} \phi_{, x z}^{p}=\delta_{1}^{2} \ddot{u}^{p} \\
c_{3} u_{, x z}^{p}+c_{2} w_{, x x}^{p}+c_{1} w_{, z z}^{p}+e_{2} \phi_{, x x}^{p}+\phi_{, z z}^{p}=\delta_{1}^{2} \ddot{w}^{p} \\
e_{1} u_{, x z}^{p}+e_{2} w_{, x x}^{p}+w_{, z z}^{p}-\eta_{3} \bar{\varepsilon} \phi_{, x x}^{p}-\eta_{3} \phi_{, z z}^{p}=0
\end{gathered}
$$

\section{Formal Solution of the Problem}

In order to facilitate solution in semiconductor medium, we introduce the scalar and vector point potential functions $\phi^{s}$ and $\psi^{s}$ through the relations

$$
u^{s}=\frac{\partial \phi^{s}}{\partial x}+\frac{\partial \psi^{s}}{\partial z}, w^{s}=\frac{\partial \varphi^{s}}{\partial z}-\frac{\partial \psi^{s}}{\partial x}
$$

Upon using relations (15) in Equations (10)-(11), we obtain

$$
\begin{gathered}
\nabla^{2} \phi^{s}-N-\ddot{\phi}^{s}=0 \\
\nabla^{2} \psi^{s}=\frac{\ddot{\psi}^{s}}{\delta^{2}} \\
\nabla^{2} N-\left[-\frac{1}{t_{n}^{+}}+\left(1+\frac{t^{n}}{t_{n}^{+}}\right) \frac{\partial}{\partial t}+t^{n} \frac{\partial^{2}}{\partial t^{2}}\right] N-\varepsilon_{n} \nabla^{2} \dot{\phi}^{s}=0
\end{gathered}
$$

The Equation (17) corresponds to purely transverse wave in the semiconductor which get decoupled from rest of the motion and not affected by the charge carrier fields.
We consider the case of time harmonic plane waves and assume wave solution of the form

$$
\begin{aligned}
& \left(\psi^{s}, \phi^{s}, N, u^{p}, w^{p}, \phi^{p}\right) \\
& =\left(\bar{\psi}^{s}, \bar{\phi}^{s}, \bar{N}, \bar{u}^{p}, \bar{w}^{p}, \bar{\phi}^{p}\right) \exp \{i k(x-c t)\}
\end{aligned}
$$

where $c=\frac{\omega}{k}$ is the phase velocity, $k$ and $\omega$ are the wave number and angular frequency of the waves respectively. Upon using solution (19) in Equations (16) to (18) and (12) to (14), the straightforward algebraic simplification leads to the following formal solution which satisfies the radiation condition in both the media:

1) Semiconductor halfspace $z \leq 0$ :

$$
\begin{gathered}
\psi^{s}=A_{3}^{s} e^{\beta z} \exp \{i k(x-c t)\} \\
\left(\phi^{s}, N\right)=\sum_{i=1}^{2}\left(1, S_{i}\right) A_{i}^{s} e^{n_{i} z} \exp \{i k(x-c t)\}
\end{gathered}
$$

2) Piezoelectric halfspace $z \geq 0$ :

$$
\left(u^{p}, w^{p}, \phi^{p}\right)=\sum_{i=1}^{3}\left(1, M_{i}, P_{i}\right) A_{i}^{p} \exp \left\{-m_{i} z+i k(x-c t)\right\}
$$

where

$$
\begin{aligned}
S_{i}= & n_{i}^{2}-\alpha^{2} \\
M_{i}= & \frac{i k m_{i}\left\{c_{3} \eta_{3}\left(m_{i}^{2}-k^{2} \bar{\varepsilon}\right)+e_{1}\left(m_{i}^{2}-k^{2} e_{2}\right)\right\}}{\left(c_{1} m_{i}^{2}-k^{2} c_{2}+\delta_{1}^{2} k^{2} c^{2}\right)\left(m_{i}^{2}-k^{2} \bar{\varepsilon}\right) \eta_{3}+\left(m_{i}^{2}-k^{2} e_{2}\right)^{2}} \\
P_{i}= & \frac{-i k e_{1} m_{i}}{\eta_{3}\left(m_{i}^{2}-k^{2} \bar{\varepsilon}\right)}+\frac{\left(m_{i}^{2}-k^{2} e_{2}\right)}{\eta_{3}\left(m_{i}^{2}-k^{2} \bar{\varepsilon}\right)} M_{i} \\
& \alpha^{2}=k^{2}\left(1-c^{2}\right), \beta^{2}=k^{2}\left(1-\frac{c^{2}}{\delta^{2}}\right), n_{i}^{2}=k^{2}\left(1-c^{2} a_{i}^{2}\right), \\
& i=1,2
\end{aligned}
$$

Here the quantities $a_{i}^{2}(i=1,2)$ and $m_{i}^{2}(i=1,2,3)$ are given by:

$$
\begin{gathered}
a_{1}^{2}+a_{2}^{2}=1+t^{n}+i \omega^{-1}\left(1+\varepsilon_{n}-\frac{t^{n}}{t_{n}^{+}}\right)+\frac{1}{\omega^{2} t_{n}^{+}} \\
a_{1}^{2} a_{2}^{2}=t^{n}+i \omega^{-1}\left(1-\frac{t^{n}}{t_{n}^{+}}\right)+\frac{1}{\omega^{2} t_{n}^{+}} \\
\sum m_{1}^{2}=k^{2} \frac{c_{2} A+\xi\left(1+\eta_{3} c_{1}\right)-c_{3}\left(c_{3} \eta_{3}+2 e_{1}\right)+c_{1} e_{1}^{2}}{c_{2}\left(1+\eta_{3} c_{1}\right)} \\
\sum m_{1}^{2} m_{2}^{2}=k^{4} \frac{c_{2} B+\xi A-c_{3}\left(c_{2} \eta_{3} \bar{\varepsilon}+2 e_{1} e_{2}\right)+e_{1}^{2}\left(c_{2}-\delta_{1}^{2} c^{2}\right)}{c_{2}\left(1+\eta_{3} c_{1}\right)} \\
m_{1}^{2} m_{2}^{2} m_{3}^{2}=k^{6} \frac{\xi B}{c_{2}\left(1+\eta_{3} c_{1}\right)} \\
\xi=\left(1-\delta_{1}^{2} c^{2}\right), \\
A=\left(c_{1} \bar{\varepsilon}+c_{2}-\delta_{1}^{2} c^{2}\right) \eta_{3}+2 e_{2}, \\
B=\left(c_{2}-\delta_{1}^{2} c^{2}\right) \eta_{3} \bar{\varepsilon}+e_{2}^{2}
\end{gathered}
$$

Upon using the solution (20) to (22) in Equations (6) and (7) 
via quantities (9) and Equation (15), the normal stresses, shear stresses, current density and displacements for the semiconductor and piezoelectric solid are obtained as:

$$
\begin{gathered}
\tau_{z z}^{s}=\left\{p \sum_{i=1}^{2} A_{i}^{s} \mathrm{e}^{n_{i} z}-q \beta A_{3}^{s} \mathrm{e}^{\beta z}\right\} \exp \{i k(x-c t)\} \\
\tau_{x z}^{s}=\left\{q \sum_{i=1}^{2} n_{i} A_{i}^{s} \mathrm{e}^{n_{i} z}+p A_{3}^{s} \mathrm{e}^{\beta z}\right\} \exp \{i k(x-c t)\} \\
J_{z}^{s}=-\sum_{i=1}^{2} S_{i} n_{i} A_{i}^{s} \mathrm{e}^{n_{i} z} \exp \{i k(x-c t)\} \\
\left(\tau_{z z}^{p}, \tau_{x z}^{p}, D_{z}^{p}\right)=\sum_{i=1}^{3}\left(y_{i}, d_{i}, b_{i}\right) A_{i}^{p} \exp \left\{-m_{i} z+i k(x-c t)\right\} \\
u^{s}=\left\{\frac{q}{2} \sum_{i=1}^{2} A_{i}^{s} \mathrm{e}^{n_{i} z}+\beta A_{3}^{s} \mathrm{e}^{\beta z}\right\} \exp \{i k(x-c t)\} \\
w^{s}=\left\{\sum_{i=1}^{2} n_{i} A_{i}^{s} \mathrm{e}^{n_{i} z}-\frac{q}{2} A_{3}^{s} \mathrm{e}^{\beta z}\right\} \exp \{i k(x-c t)\}
\end{gathered}
$$

where

$$
\begin{aligned}
& p=\left(k^{2}+\beta^{2}\right), q=2 i k, \\
& y_{i}=\frac{\bar{\rho}}{\delta^{2} \delta_{1}^{2}}\left\{i k\left(c_{3}-c_{2}\right)-\left(c_{1} M_{i}+P_{i}\right) m_{i}\right\}, \\
& d_{i}=\frac{\bar{\rho}}{\delta^{2} \delta_{1}^{2}}\left\{\frac{c_{2}}{2}\left(i k M_{i}-m_{i}\right)+i k e_{2} P_{i}\right\}, \\
& b_{i}=i k\left(e_{1}-e_{2}\right)-m_{i}\left(M_{i}-\eta_{3} P_{i}\right), i=1,2,3
\end{aligned}
$$

and $A_{i}^{s}, A_{i}^{p}(i=1,2,3)$ are the unknowns to be determined.

\section{Secular Equation}

We obtain a system of six homogeneous algebraic equations in the six unknowns $A_{i}^{s}$ and $A_{i}^{p}(i=1,2,3)$ upon using the formal solution obtained in the previous section in the boundary conditions (8) which has a non-trivial solution if the determinant of the coefficient of $A_{i}^{s}, A_{i}^{p},(1,2,3)$ vanishes and this require lengthy algebraic reductions and simplifications which leads to the following secular equation for the propagation of guided waves in the considered composite structure

$$
\operatorname{det}\left(a_{i j}\right)=0,(i, j=1,2,3, \cdots, 6)
$$

where the non-zero elements $a_{i j}$ are given below

$$
\begin{aligned}
& a_{12}=p, a_{13}=-q \beta, a_{1 k}=y_{k}(k=4,5,6), a_{21}=q, \\
& a_{23}=p, a_{2 k}=d_{k}(k=4,5,6), a_{32}=\frac{q}{2}, a_{33}=\beta, \\
& a_{34}=a_{35}=a_{36}=1, a_{41}=1, a_{43}=-\frac{q}{2}, \\
& a_{4 k}=M_{k}(k=4,5,6), a_{51}=n_{1}+n_{2}, a_{52}=-\left(n_{1} n_{2}+\alpha^{2}\right), \\
& a_{5 k}=P_{k}(k=4,5,6), a_{61}=\frac{S_{1} n_{1}-S_{2} n_{2}}{n_{1}-n_{2}}, \\
& a_{52}=-n_{1} n_{2}\left(n_{1}+n_{2}\right), a_{6 k}=-b_{k}(k=4,5,6)
\end{aligned}
$$

The complex secular Equation (35) contains complete information about the characteristics of the waves traveling at the interface.

\section{Solution of Secular Equation}

In general, wave number and hence the phase velocities of the waves are complex quantities, therefore the waves are attenuated in space. In order to solve the secular equations, we take

$$
c^{-1}=V^{-1}+i \omega^{-1} Q
$$

where $k=R+i Q, \quad R=\frac{\omega}{V}$ and $R, Q$ are real numbers. Here, it may be noted that $V$ and $Q$ respectively, represent the phase velocity and attenuation coefficient of the waves. Upon using representation (37) in various relevant relations, the complex roots $m_{i}^{2}(i=1,2,3)$ can be computed from (26) with the help of Cardano's method. The roots $m_{i}^{2}(i=1,2,3)$ are further used to solve secular Equation (35) to obtain phase velocity $(V)$ and attenuation coefficient $(Q)$ of the surface waves by using function iteration numerical technique whose procedure is outlined by Sharma et al. (2010).

For initial value of $c=c_{0}=\left(V_{0}, Q_{0}\right)$, the roots $m_{i}(i=1,2,3)$ are computed from Equations (26) by using Cardano's method for each value of non-dimensional wave number $(R)$ for assigned frequency. The values of $m_{i}(i=1,2,3)$ so obtained are then used in secular Equation (35) to obtain the current values of $V$ and $Q$. The process is terminated as and when the condition $\left|V_{n+1}-V_{n}\right|<\varepsilon, \varepsilon$ being arbitrarily small number to be selected at random to achieve the accuracy level, is satisfied. The procedure is continuously repeated for different values of $R$ to obtain corresponding values of the $V$ and $Q$. Thus, the real phase velocity and attenuation coefficient of Rayleigh type surface waves in the composite structure under study can be computed from dispersion relation (35).

\section{Amplitudes of Field Functions}

The amplitudes of various field functions at the surface $(z=0)$ are obtained as:

$$
\begin{aligned}
& \left(u^{s}, w^{s}, N, \tau_{z z}^{s}, \tau_{x z}^{s}\right)=\left(U^{s}, W^{s}, N^{*}, \sigma_{z z}^{s}, \sigma_{x z}^{s}\right) A_{1}^{s} \exp \{i R(x-V t)\} \\
& \left(u^{p}, w^{p}, \phi^{p}, \tau_{z z}^{p}, \tau_{x z}^{p}\right)=\left(U^{p}, W^{p}, \Phi^{p}, \sigma_{z z}^{p}, \sigma_{x z}^{p}\right) A_{1}^{s} \exp \{i R(x-V t)\}
\end{aligned}
$$

where

$$
\begin{gathered}
U^{s}=\left\{\frac{q}{2}\left(1+L_{2}^{s}\right)+\beta L_{3}^{s}\right\} \exp (-Q x) \\
W^{s}=\left(n_{1}+n_{2} L_{2}^{s}-\frac{q}{2} L_{3}^{s}\right) \exp (-Q x) \\
N^{*}=\left(S_{1}+S_{2} L_{2}^{s}\right) \exp (-Q x) \\
U^{p}=\left(L_{1}^{p}+L_{2}^{p}+L_{3}^{p}\right) \exp (-Q x) \\
W^{p}=\left(M_{1} L_{1}^{p}+M_{2} L_{2}^{p}+M_{3} L_{3}^{p}\right) \exp (-Q x) \\
\Phi^{p}=\left(P_{1} L_{1}^{p}+P_{2} L_{2}^{p}+P_{3} L_{3}^{p}\right) \exp (-Q x)
\end{gathered}
$$




$$
\begin{aligned}
\sigma_{z z}^{s} & =\left\{p\left(1+L_{2}^{s}\right)-q \beta L_{3}^{s}\right\} \exp (-Q x) \\
\sigma_{x z}^{s} & =\left\{q\left(n_{1}+n_{2} L_{2}^{s}\right)+p L_{3}^{s}\right\} \exp (-Q x) \\
\sigma_{z z}^{p} & =\left(y_{1} L_{1}^{p}+y_{2} L_{2}^{p}+y_{3} L_{3}^{p}\right) \exp (-Q x) \\
\sigma_{x z}^{p} & =\left(d_{1} L_{1}^{p}+d_{2} L_{2}^{p}+d_{3} L_{3}^{p}\right) \exp (-Q x)
\end{aligned}
$$

Here

$$
\begin{aligned}
& L_{2}^{s}=-\frac{\Delta_{i j}^{2 s}}{\Delta_{i j}^{0}}, L_{3}^{s}=\frac{\left(y_{2}-y_{3}\right) \Delta_{i j}^{3 s}}{\Delta_{i j}^{0}}, L_{1}^{p}=-\frac{\left(y_{2}-y_{3}\right) \Delta_{i j}^{1 p}}{\Delta_{i j}^{0}}, \\
& L_{2}^{p}=\frac{\left(y_{2}-y_{3}\right)^{2} \Delta_{i j}^{2 p}}{\left(y_{1}-y_{3}\right) \Delta_{i j}^{0}}, L_{3}^{p}=\frac{\left(y_{2}-y_{3}\right)^{2} \Delta_{i j}^{3 p}}{\left(y_{2}-y_{1}\right) \Delta_{i j}^{0}}
\end{aligned}
$$

The elements of matrices $\Delta_{i j}^{0}, \Delta_{i j}^{2 s}, \Delta_{i j}^{3 s}, \Delta_{i j}^{1 p}, \Delta_{i j}^{2 p}$ and $\Delta_{i j}^{3 p}$ are defined in appendix.

\section{Numerical Results and Discussion}

In order to illustrate the analytical developments in the previous section, we now perform some numerical computations and simulations. The composite material chosen for the purpose of numerical calculations is composed of $6 \mathrm{~mm}$ class cadmium selenide (CdSe) piezoelectric material and n-type silicon ( $\mathrm{Si}$ ) semiconductor. The physical data for piezoelectric and semiconductor half spaces are given as under:

1) Piezoelectric half space [Sharma and Pal (2004)]:

$$
\begin{aligned}
& c_{11}=7.41 \times 10^{10} \mathrm{~nm}^{-2}, c_{13}=3.93 \times 10^{10} \mathrm{~nm}^{-2}, \\
& c_{33}=8.36 \times 10^{10} \mathrm{~nm}^{-2}, c_{44}=1.32 \times 10^{10} \mathrm{~nm}^{-2}, \\
& e_{31}=-0.160 \mathrm{~cm}^{-2}, e_{33}=0.347 \mathrm{~cm}^{-2}, \\
& e_{15}=-0.138 \mathrm{~cm}^{-2}, \varepsilon_{11}=8.26 \times 10^{11} \mathrm{C} 2 \mathrm{~N}-1 m^{-2}, \\
& \varepsilon_{33}=9.03 \times 10^{11} \mathrm{C} 2 \mathrm{~N}-1 \mathrm{~m}^{-2}, \rho^{p}=5504 \mathrm{kgm}^{-3} .
\end{aligned}
$$

2) Semiconductor halfspace [Sharma et al. (2007)]:

$$
\begin{aligned}
& \lambda=0.64 \times 10^{11} \mathrm{~nm}^{-2}, \mu=0.65 .0 \mathrm{~nm}^{-2}, \\
& D^{n}=0.35 \times 10^{-2} \mathrm{~m}^{-2} \mathrm{~s}^{-1}, n_{0}=10^{20} \mathrm{~m}^{-3}, \\
& \alpha_{T}=2.6 \times 10^{-6} \mathrm{~K}^{-1}, \rho^{s}=2300 \mathrm{~kg} \cdot \mathrm{m}^{-3} .
\end{aligned}
$$

Here we present the effect of different interacting fields and corresponding parameters on the surface wave at the interface of considered structure. The profiles are plotted with respect to non-dimensional wave number $(R)$ on linear-log scales. The corresponding results in the physical domain can be obtained with the help of quantities defined in Equation (9) from the instant non-dimensional one. The numerical computations have been performed; correct upto four decimal places here, by employing the procedure outlined in section (Solution of Secular Equation) by using MATLAB programming. The computer simulated results have been presented graphically in Figures 2 to 9.

Figure 2 represents the variations of longitudinal and transverse displacements versus distance $(x)$ for semiconductor halfspace in the considered composite. The profiles show that as we move along the direction of wave propagation, the displacements of the particles of the medium decreases and ulti- mately vanish at some distance. Moreover the magnitude of the longitudinal displacement is higher than that of transverse displacement. The magnitude decreases because of the resistance offered by the medium to the wave propagation due to the anelastic properties of the materials, in which the energy of the elastic wave is lost to heat the material by causing permanent deformations.

Figure 3 presents the variations of the longitudinal and transverse displacements for the piezoelectric halfspace versus distance in the composite structure. Here we have found the similar profiles as in case of silicon halfspace in the considered composite, which justify the boundary conditions which require that the respective displacements in both the materials must balance the effect of each other at the interface in order to stabilize the welded contact at $z=0$, otherwise such structure is impossible to exist.

The Figure 4 displays the variations of the carrier concentration at the interface of composite with the distance. It is found

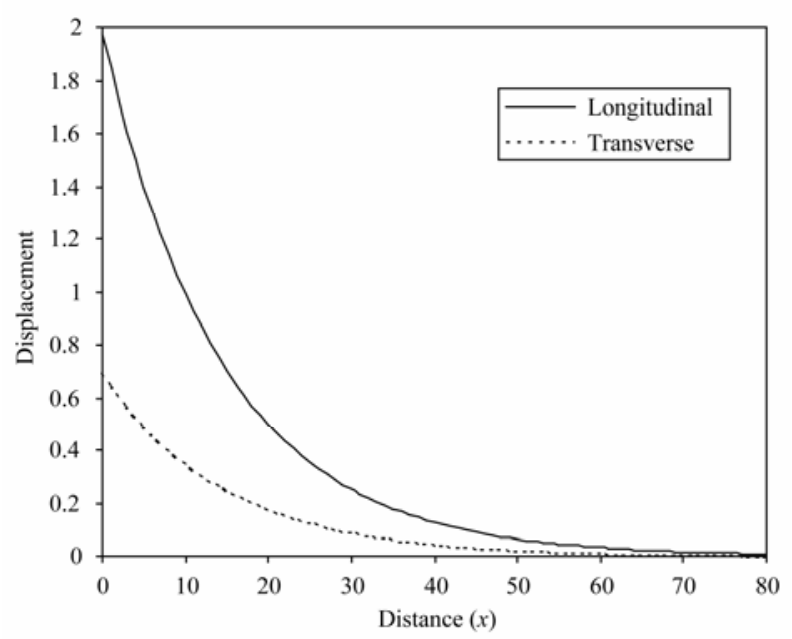

Figure 2.

Variations of displacements for semiconductor halfspace versus distance.

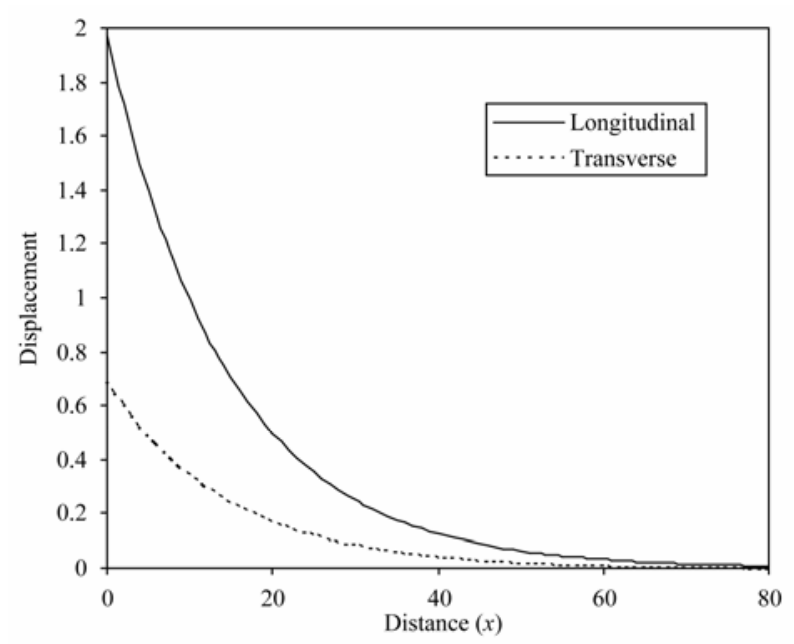

Figure 3.

Variations of displacements for piezoelectric halfspace versus distance. 
that the change in the carrier concentration also decreases with distance, before it ultimately vanishes after some distance along the direction of wave propagation.

In Figure 5 the variations of piezoelectric potential are plotted with the distance, which also follow the similar trend as that of the carrier concentration. It also justifies the boundary condition that change in carrier concentration balance the change in piezoelectric potential at the interface. The disturbance causes a surface acoustic at piezoelectric halfspace which is associated with an electric field. This electric field changes the carrier concentration at the interface as the negatively charged electrons interact with it. In this process the carriers follow the electric field associated with the surface acoustic wave and acquire energy from this electric field.

Figure 6 shows the variations of normal and shear stresses for the semiconductor halfspace versus distance in the composite structure. It is observed that both the stresses decrease with the increase in the distance along the direction of wave propa-

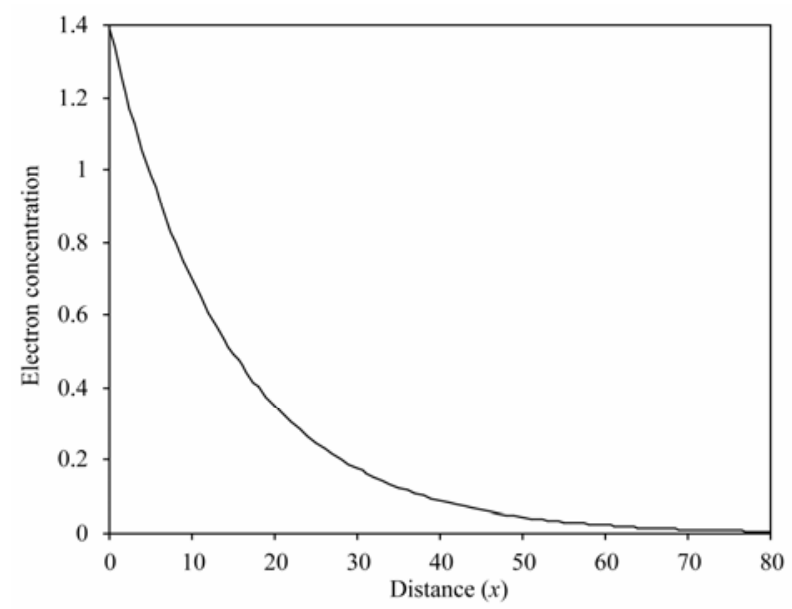

Figure 4.

Variations of electron concentration for semiconductor halfspace versus distance.

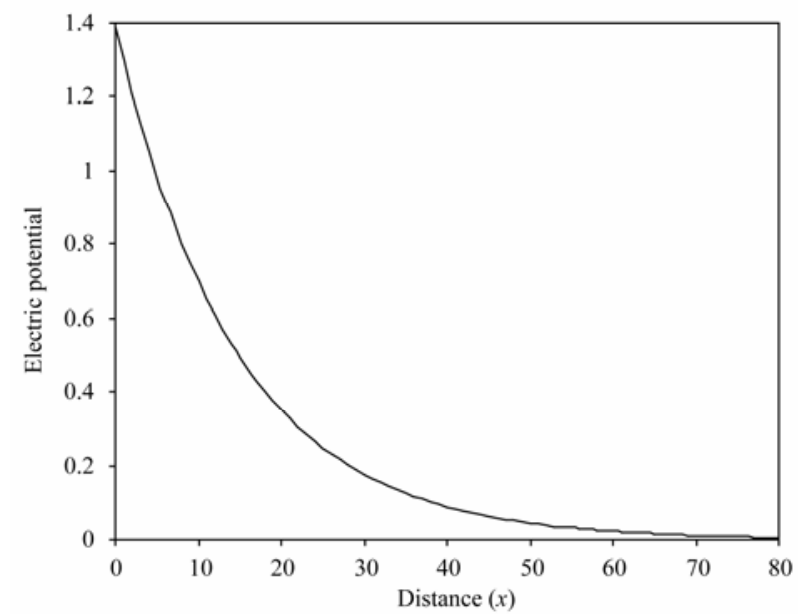

Figure 5.

Variations of electric potential for piezoelectric halfspace versus distance. gation. The shear stress possesses higher magnitude than that of the normal stress. In Figure 7, the variations of normal and shear stresses for piezoelectric halfspace in the same composite are plotted. We found the similar profiles with equal magnitudes and same vanishing distance along the direction of wave propagation as in case of semiconductor halfspace. The shear stress possesses larger magnitude in comparison to the normal stress in both the material components of the composite. The results show that at the interface the stresses balance the effect of each other.

Figure 8 displays the variations of phase velocity with the wave number at the interface of the composite. The profiles are noticed to be clearly dispersive, hence showing that phase velocity is dependant on the wavelength of the wave. Phase velocity possesses large magnitude at long wavelengths in comparison to small wavelengths. This is due to the reason that long wavelengths penetrate the medium to a greater extent thereby brings the various coupling field in to play which con-

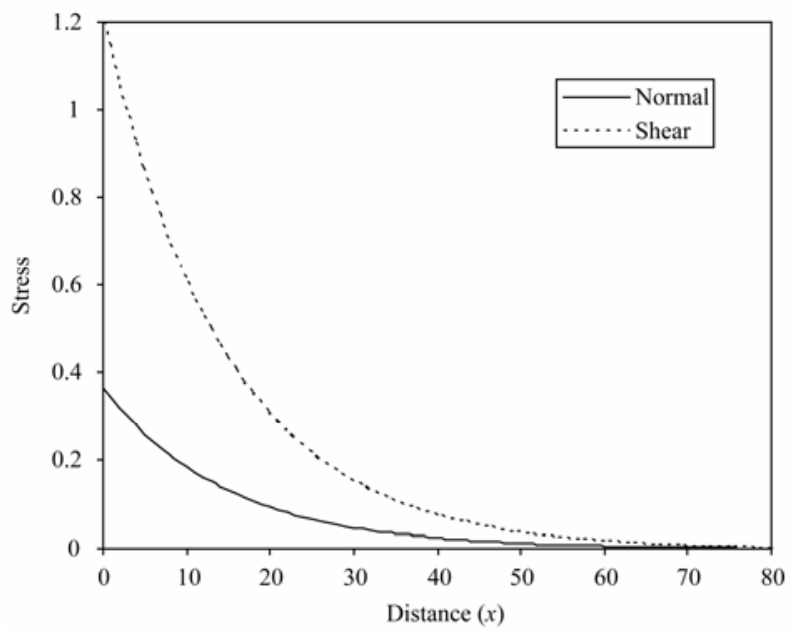

Figure 6.

Variations of stresses for semiconductor halfspace versus distance.

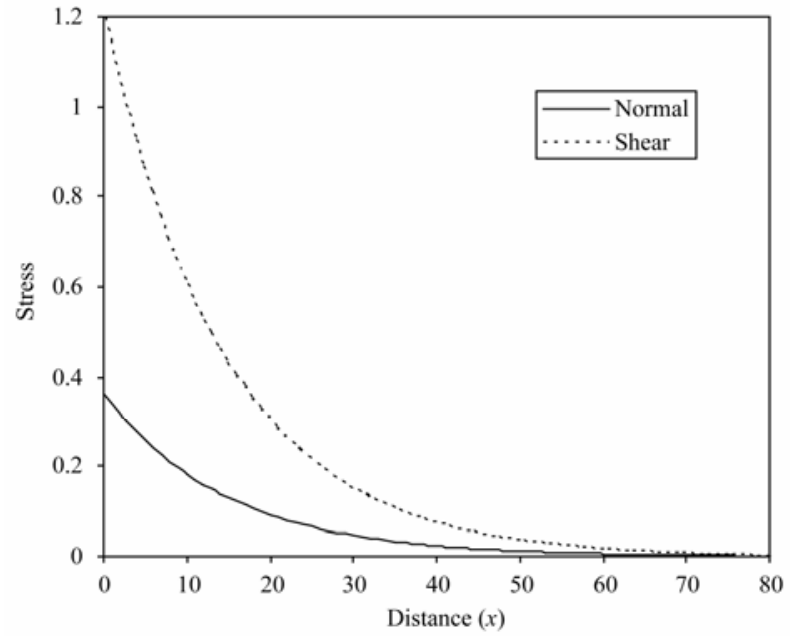

Figure 7.

Variations of stresses for piezoelectric halfspace versus distance. 
tribute to increase the phase velocity. The magnitude of phase velocity decreases with decrease in the life time of the carrier field.

Figure 9 shows the variations of attenuation coefficient with the wave number at the interface of the composite. The attenuation increases with decreasing wavelength. It is also noticed that it decreases with decreasing life time of the carrier field.

\section{Concluding Remarks}

1) The functional iteration method along with the Cardano method has been successfully employed to solve complex characteristic equations to obtain the surface waves characteristics at the interface of composite.

2) At the interface of the considered composite the displacements, stresses, electron concentration, electric potential decrease along the direction of wave propagation and then vanish after some distance.

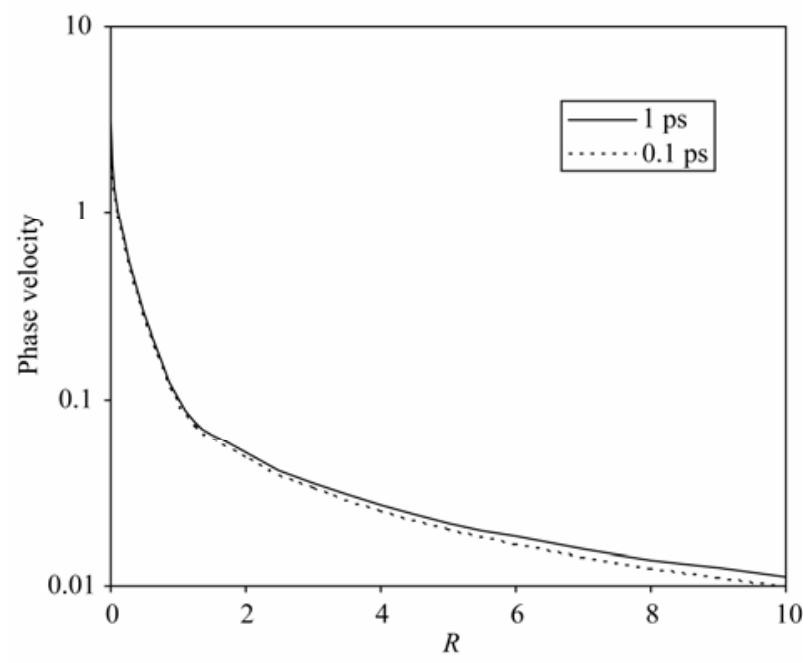

Figure 8.

Variations of phase velocity versus wave number.

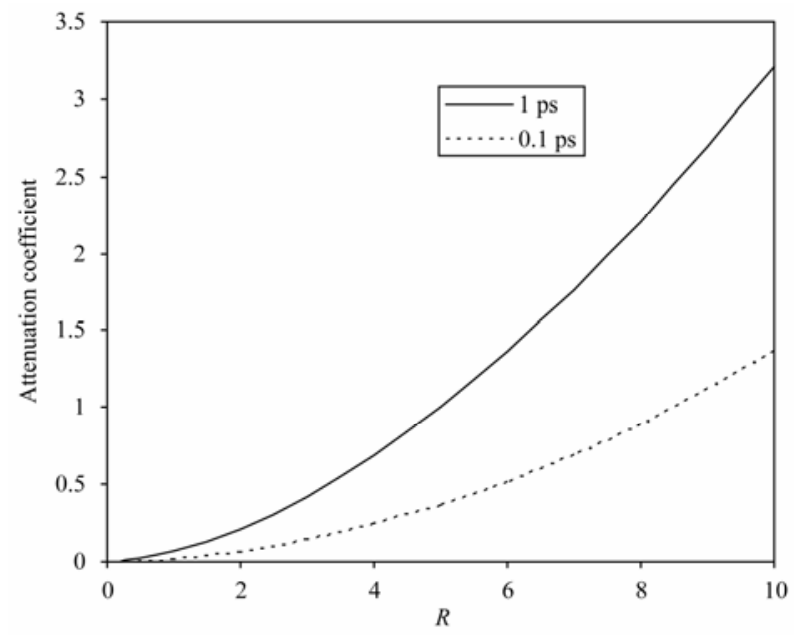

Figure 9.

Variations of attenuation coefficient versus wave number.
3) The phase velocity possesses large magnitude at long wavelengths which goes on decreasing with the decreasing wavelength hence showing a dispersive character.

4) The attenuation increases with the decreasing wavelength in the considered composite structure.

5) The phase velocity as well as attenuation decreases with decreasing life time of the carrier field.

6) The study may find applications in fabrication of micro-electromechanical surface acoustic wave devices.

\section{Acknowledgements}

The authors are thankful to the reviewers for their deep interest and useful suggestions for the improvement of this work.

\section{References}

Bleustein, J. L. (1968). A new surface wave in piezoelectric materials. Applied Physics Letters, 13, 412-414. doi:10.1063/1.1652495

Collins, J. H., Lakin, K. M., Quate, C. F., \& Shaw, H. J. (1968). Amplification of acoustic surface waves with adjacent semiconductor and piezoelectric crystals. Applied Physics Letters, 13, 314-316. doi: $10.1063 / 1.1652628$

Curie, J., \& Curie, P. (1880). Development par compression de l'etricite polaire das les cristaux hemledres a faces inclines. Bulletin No. 4 de la Societee Minearalogique de France 3.

de Lorenzi, H. G., \& Tierten, H. F. (1975). On the interaction of the electromagnetic field with heat conducting deformable semiconductors. Journal of Mathematical Physics, 16, 938-957. doi:10.1063/1.522600

Dietz, D. R., Busse, L. J., \& Fife M. J. (1988). Acoustoelectric detection of ultrasound power with composite piezoelectric and semiconductor devices. IEEE Transactions on Ultrasonics, Ferroelectrics and Frequency Control, 35, 146-151.

doi:10.1109/58.4164

Fischler, C. (1970). Acoustoelectric Amplification in composite Piezoelectric and Semiconducting structures. IEEE Transactions on Electron Devices, 17, 214-218. doi:10.1109/T-ED.1970.16956

Gulyaev, Y. V. (1969). Electroacoustic surface waves in solids. Soviet Physics JEPT Letters, 9, 37-38.

Hutson, A. R., \& White, D. L. (1962). Elastic wave propagation in piezoelectric semiconductors. Journal of Applied Physics, 33, 40-47. doi:10.1063/1.1728525

Ingebrigtsen, K. A. (1970). Linear and nonlinear attenuation of acoustic surface waves in a piezoelectric coated with a semiconductor film. Journal of Applied Physics, 41, 454-459. doi:10.1063/1.1658696

Jin, J., Wang, Q., \& Quek, S. T. (2002). Lamb wave propagation in a metallic semi-infinite medium covered with piezoelectric layer. International Journal of Solids Structures, 39, 2547-2556.

Kagan, V. D. (1997). Propagation of a surface acoustic wave in a layered system containing a two dimensional conducting layer. Semiconductors, 31, 407-410. doi:10.1134/1.1187321

Kleinert, P., García-Cristóbal, A., \& Santos, P. V. (2005). Surface acoustic-waves-induced space-charge waves in electron-hole systems. Journal of Solid State Communications, 34, 535-539. doi:10.1016/j.ssc.2005.02.039

Maruszewski, B. (1989). Thermodiffusive surface waves in semiconductors. Journal of Acoustic Society of America, 85, 1967-1977. doi:10.1121/1.397850

Maugin, G. A., \& Daher, N. (1986). Phenomenological theory of elastic semiconductors. International Journal of Engineering Sciences, 24 703-731. doi:10.1016/0020-7225(86)90106-0

Parmenter, R. H. (1953). The acousto-electric effect. Physical Reveiw, 89, 990-998. doi:10.1103/PhysRev.89.990

Sharma, J. N., \& Pal, M. (2004). Propagation of lamb waves in a trans- 
versely isotropic piezothermoelastic plate. Journal of Sound and Vibration, 270, 587-610. doi:10.1016/S0022-460X(03)00093-2

Sharma, J. N., Pal, M., \& Chand, D. (2005). Propagation characteristics of Rayleigh waves in transversely isotropic piezothermoelastic materials. Journal of Sound and Vibration, 284, 227-248. doi:10.1016/j.jsv.2004.06.036

Sharma, J. N., Sharma, I., \& Chand, S. (2008). Elasto-thermodiffusive surface waves in a semiconductor half-space underlying a fluid with varying temperature. Journal of Thermal Stresses, 31, 956-975. doi:10.1080/01495730802250524

Sharma, J. N., Sharma, K. K., \& Kumar, A. (2010). Surface waves in a piezoelectric-semiconductor composite structure. International Journal of Solids and Structures, 47, 816-826. doi:10.1080/01495730802250524

Sharma, J. N., \& Thakur, N. (2006). Plane harmonic elasto-thermodiffusive waves in semiconductor materials. Journal of Mechanics of Materials and Structures, 1, 813-835. doi:10.2140/jomms.2006.1.813

Sharma, J. N., Thakur, N., \& Singh, S. (2007). Propagation characteristics of elasto-thermodiffusive surface waves in semiconductor material half space. Journal of Thermal Stresses, 30, 357-380. doi:10.1080/01495730601146311

Sharma, J. N., Thakur, N., \& Singh, S. (2009). Elasto-thermodiffusive (ETNP) surface waves in semiconductor materials. International

\section{Appendix}

The elements of $\Delta_{i j}^{0}, \Delta_{i j}^{2 s}, \Delta_{i j}^{3 s}, \Delta_{i j}^{1 p}, \Delta_{i j}^{2 p}$ and $\Delta_{i j}^{3 p}$ are given by

$$
\begin{gathered}
\Delta_{11}^{0}=q\left(n_{2}-\frac{d_{3}}{2}\right)\left(y_{2}-y_{3}\right)-\left(p-\frac{q y_{3}}{2}\right)\left(d_{2}-d_{3}\right), \\
\Delta_{12}^{0}=\left(\beta d_{3}-p\right)\left(y_{2}-y_{3}\right)-q\left(\beta+y_{3}\right)\left(d_{2}-d_{3}\right), \\
\Delta_{13}^{0}=\left(d_{1}-d_{3}\right)\left(y_{2}-y_{3}\right)-\left(y_{1}-y_{3}\right)\left(d_{2}-d_{3}\right), \\
\Delta_{21}^{0}=\left(n_{2}-\frac{q}{2} M_{3}\right)\left(y_{2}-y_{3}\right)-\left(p-\frac{q}{2} y_{3}\right)\left(M_{2}-M_{3}\right), \\
\Delta_{22}^{0}=\left(\frac{q}{2}+\beta M_{3}\right)\left(y_{2}-y_{3}\right)-\beta\left(q+y_{3}\right)\left(M_{2}-M_{3}\right), \\
\Delta_{23}^{0}=\left(M_{1}-M_{3}\right)\left(y_{2}-y_{3}\right)-\left(y_{1}-y_{3}\right)\left(M_{2}-M_{3}\right), \\
\Delta_{31}^{0}=\left(S_{2}-\frac{q}{2} P_{3}\right)\left(y_{2}-y_{3}\right)-\left(p-\frac{q}{2} y_{3}\right)\left(P_{2}-P_{3}\right), \\
\Delta_{32}^{0}=P_{3}\left(y_{2}-y_{3}\right)-\beta\left(q+y_{3}\right)\left(P_{2}-P_{3}\right), \\
\Delta_{33}^{0}=\left(P_{1}-P_{3}\right)\left(y_{2}-y_{3}\right)-\left(y_{1}-y_{3}\right)\left(P_{2}-P_{3}\right),
\end{gathered}
$$

The quantities $\Delta_{i j}^{2 s}$ can be obtained from $\Delta_{i j}^{0}$ by changing $n_{2}$ by $n_{1}, n_{1}$ by $n_{2}$ and $S_{2}$ by $S_{1}$.
Journal of Solids and Structures, 46, 2309-2319. doi:10.1016/j.ijsolstr.2009.01.019

Sharma, J. N., \& Walia, V. (2007). Further Investigations on Rayleigh waves in piezothermoelastic materials. Journal of Sound and Vibration, 301, 189-206. doi:10.1016/j.jsv.2006.09.018

Tien, P. K. (1968). Nonlinear theory of ultrasonic wave amplification and current saturation in piezoelectric semiconductors. Physical Review, 171, 970-986. doi:10.1103/PhysRev. 171.970

Wang, Q. (2002). Wave propagation in a piezoelectric coupled solid medium. Journal of Applied Mechanics, 69, 819-824. doi:10.1115/1.1488662

Weinreich, G., Sanders, Jr T. M., and White, H. G. (1959). Acoustoelectric effect n-type Germanium. Physical Reveiw, 114, 33-44. doi:10.1103/PhysRev.114.33

White, D. L. (1962). Amplification of ultrasonic waves in piezoelectric semiconductors. Journal of Applied Physics, 33, 2547-2554. doi:10.1063/1.1729015

Yang, J. S., \& Zhou, H. G. (2005). Propagation and amplification of gap waves between a piezoelectric halfspace and a semiconductor film. Acta Mechanica, 176, 83-93. doi:10.1063/1.1729015

Yang, J. S., \& Zhou, H. G. (2005). Amplification of acoustic waves in piezoelectric semiconductor plates. International Journal of Solids Structures, 42, 3171-3183. doi:10.1016/j.ijsolstr.2004.10.011

$$
\begin{gathered}
\Delta_{11}^{3 s}=2 i k \delta^{2}, \quad \Delta_{12}^{3 s}=\Delta_{11}^{2 s}, \quad \Delta_{13}^{3 s}=\Delta_{13}^{2 s}, \quad \Delta_{21}^{3 s}=1, \quad \Delta_{22}^{3 s}=\Delta_{21}^{2 s}, \\
\Delta_{23}^{3 s}=\Delta_{23}^{2 s}, \quad \Delta_{31}^{3 s}=n_{1}+n_{2}, \quad \Delta_{32}^{3 s}=\Delta_{31}^{2 s}, \quad \Delta_{33}^{3 s}=\Delta_{33}^{2 s}, \quad \Delta_{11}^{1 p}=\Delta_{31}^{3 s}, \\
\Delta_{12}^{1 p}=\left(p-\frac{q}{2} y_{2}\right)\left(d_{2}-d_{3}\right)-q\left(n_{1}-\frac{q}{2} d_{2}\right)\left(y_{2}-y_{3}\right), \\
\Delta_{13}^{1 p}=q\left(\beta+y_{2}\right)\left(d_{2}-d_{3}\right)-\left(\beta d_{2}-p\right)\left(y_{2}-y_{3}\right), \\
\Delta_{22}^{1 p}=\left(p-\frac{q}{2} y_{2}\right)\left(M_{2}-M_{3}\right)-\left(n_{1}-\frac{q}{2} M_{2}\right)\left(y_{2}-y_{3}\right), \\
\Delta_{23}^{1 p}=\beta\left(q+y_{2}\right)\left(M_{2}-M_{3}\right)-\left(\frac{q}{2}+\beta M_{2}\right)\left(y_{2}-y_{3}\right), \\
\Delta_{31}^{1 p}=\Delta_{31}^{3 s}, \quad \Delta_{32}^{1 p}=\left(p-\frac{q}{2} y_{2}\right)\left(P_{2}-P_{3}\right)-\left(S_{1}-\frac{q}{2} P_{2}\right)\left(y_{2}-y_{3}\right), \\
\Delta_{33}^{1 p}=\beta\left(q+y_{2}\right)\left(P_{2}-P_{3}\right)-P_{2}\left(y_{2}-y_{3}\right),
\end{gathered}
$$

The expressions for $\Delta_{i j}^{2 p}$ and $\Delta_{i j}^{3 p}$ can be written from those of $\Delta_{i j}^{1 p}$ by cyclic permuting the suffixes of quantities $y_{i}$, $d_{i}, P_{i}$, and $M_{i}$ cyclically. 